\title{
Magnetic resonance imaging of the brain in survivors of childhood acute lymphoblastic leukemia
}

\author{
MOHAMED AHMED BADR ${ }^{1}$, TAMER HASAN HASSAN ${ }^{1}$, KHALED MOHAMED EL-GERBY $^{2}$ \\ and MOHAMED EL-SAYED LAMEY ${ }^{1}$
}

Departments of ${ }^{1}$ Pediatrics and ${ }^{2}$ Radiodiagnosis, Zagazig University, Zagazig, Egypt

Received August 29, 2012; Accepted November 2, 2012

DOI: $10.3892 / \mathrm{ol} .2012 .1072$

\begin{abstract}
The issue of delayed neurological damage as a result of treatment is becoming increasingly important now that an increased number of children survive treatment for acute lymphoblastic leukemia (ALL). Following modification of the treatment protocols, severe symptomatic late effects are rare, and most adverse effects are detected by sensitive imaging methods such as magnetic resonance imaging (MRI) or by neuropsychological testing. In this study we aimed to determine the prevalence and characteristics of late central nervous system (CNS) damage by MRI and clinical examination in children treated for ALL. A cross-sectional study was carried out at the pediatric oncology unit of Zagazig University, Egypt, and included 25 patients who were consecutively enrolled and treated according to the modified Children's Cancer Group (CCG) 1991 protocol for standard risk ALL and the modified CCG 1961 protocol for high-risk ALL and who had survived more than 5 years from the diagnosis. All relevant data were collected from patients' medical records; particularly the data concerning the initial clinical presentation and initial brain imaging. All patients were subjected to thorough history and full physical examination with special emphasis on the neurological system. MRI of the brain was performed for all patients. The mean age of patients was $6.9 \pm 3.04$ years at diagnosis and was $12.9 \pm 3.2$ years at the time of study. The patients comprised 14 boys and 11 girls. Abnormal MRI findings were detected in six patients (24\%). They were in the form of leukoencephalopathy in two patients $(8 \%)$, brain atrophy in two patients $(8 \%)$, old infarct in one patient $(4 \%)$ and old hemorrhage in one patient (4\%). The number of abnormal MRI findings was significantly higher in high-risk patients, patients who had CNS manifestations at diagnosis and patients who had received cranial irradiation. We concluded that cranial irradiation is associated with higher incidence of MRI changes
\end{abstract}

Correspondence to: Dr Tamer Hasan Hassan, Department of Pediatrics, Zagazig University, Borg Al Salim 3 Qaumia Street, Sharkia, Zagazig 44111, Egypt

E-mail:dr.tamerhassan@yahoo.com

Key words: acute lymphoblastic leukemia, magnetic resonance imaging, brain, survivors in children treated for ALL. Limitation of cranial irradiation to selected patients contributed to a lower incidence of neurological complications in our study. MRI is a sensitive radiological tool to detect structural changes in children treated for ALL, even in asymptomatic cases.

\section{Introduction}

Acute lymphoblastic leukemia (ALL) is the most common malignancy in children. It accounts for approximately $25 \%$ of all childhood cancers and almost $75 \%$ of childhood leukemias. Treatment results in childhood ALL are one of the true success stories of modern clinical oncology with an overall cure rate currently approaching more than $85 \%$ in the developed world, mainly through the application of intensive multi-agent chemotherapeutic regimens $(1,2)$.

This therapeutic progress is the result of treatment advances that began with the identification of effective single agent chemotherapy in the late 1940 s, followed by development of combination chemotherapy and maintenance chemotherapy in the 1950s and early 1960s and the implementation of effective central nervous system (CNS) preventive therapy in the 1960s and 1970s (3).

CNS-directed therapy is a key contributing factor to improving survival among children with ALL. When cranial radiation was linked to neurocognitive deficits, therapeutic regimens were modified to reduce or eliminate cranial radiation and substituted it with intensified intrathecal and systemic chemotherapy. These CNS-directed therapies could also influence the risk of late neurological outcomes (4).

Neurological complications are common, both during and following completion of therapy (5). Common neurological complications developing after completion of ALL treatment include leukoencephalopathy and neurocognitive defects (6).

Several studies have used magnetic resonance imaging (MRI) to detect neurologic complications in patients treated for ALL. A wide range of results have been reported $(7,8)$.

Hemosiderin and white matter lesions are two of the most common neurological complications found on MRI that may be related to cranial irradiation and intrathecal methotrexate (MTX) therapy in childhood ALL (5).

We aimed to determine the prevalence and characteristics of late CNS damage by MRI and clinical examination in children treated for ALL. 


\section{Materials and methods}

Patients. This study was carried out at the outpatient clinic of the Pediatric Oncology Unit of Zagazig University Hospital and the MRI Unit of the Radiodiagnosis Department of Zagazig University between September 2010 and August 2011. It included 25 patients who were consecutively enrolled and treated according to the modified Children's Cancer Group (CCG) 1991 protocol for standard risk ALL and modified CCG 1961 protocol for high-risk ALL and who had survived more than 5 years from the diagnosis. The modified CCG 1991 protocol for standard risk ALL and modified CCG 1961 protocol for high-risk ALL have been applied as a unified protocol in Egypt since 2004.

All relevant data were collected from patients' medical records, specifically those concerning the initial clinical presentation and initial brain imaging.

All patients were subjected to: i) Thorough history and full physical examination with special emphasis on the neurological system; ii) MRI of the brain using Philips Achieva class II MRI 1.5-T scanner (Philips Medical Systems, Best, The Netherlands) using T1-weighted (T1W) sagittal spin-echo [repetition time (TR), $500 \mathrm{msec}$; echo time (TE), $15 \mathrm{msec}$ ], T2-weighted transverse fast spin-echo (TR, 3,300 msec; TE, $100 \mathrm{msec}$ ), GE transverse (TR, $300 \mathrm{msec}$; TE, $30 \mathrm{msec}$; flip angle, $30^{\circ}$ ), and fluid attenuated inversion recovery (FLAIR) coronal (TR, 8,000 msec; TE, $110 \mathrm{msec}$; T1, 2,400 msec) sequences.

\section{Definitions of abnormal MRI findings}

Leukoencephalopathy. Hyperintense white matter abnormalities were graded according to a modification of the system of Wilson et al, 1991. Grade I was defined as patchy, mildly increased signal intensity in the periventricular white matter, grade II as moderate changes that extend almost to the graywhite junction, sparing the subcortical U-fibers, and grade 3 as severe changes, confluent from the level of the frontal horns to that of the trigones, with or without involvement of the U-fibers.

Brain atrophy. The definition was based on visual evaluation of the width of cortical sulci and the size of the ventricles and was divided into three grades (mild, moderate and severe).

Old infarcts. Old infarcts were diagnosed by the detection of brain parenchymal loss pertaining to arterial territory or discrete lesions with hypointensity on T1-weighted images and hyperintensity on $\mathrm{T} 2$-weighted images.

Old hemorrhages. Old hemorrhages were defined as focal rounded areas of very low signal intensity (attributable to the presence of hemosiderin) detected in any part of the brain.

Summary of modified CCG 1991 protocol for standard risk ALL. Patients were eligible for this protocol if they had previously untreated ALL with $>25 \%$ blasts (L1 or L2 morphology) in bone marrow, age 1-9.99 years and initial WBC $<50.000 / \mu 1$. Patients with CNS disease at diagnosis, overt testicular leukemia, FAB L3 and T-cell ALL (T-ALL) were not eligible.

Patients received induction chemotherapy for one month (i.v. vincristine, p.o. dexamethasone, i.m. L-asparaginase, i.t. MTX and i.t. Ara-C), consolidation therapy for 4 weeks (i.v. vincristine, p.o. 6-mercaptopurine and i.t. MTX), interim maintenance I for 2 months (i.v. vincristine, p.o. dexamethasone, p.o. 6-mercaptopurine, p.o. MTX and i.t. MTX), delayed intensification for 2 months (i.v. vincristine, i.v. doxorubicin, p.o. dexamethasone, i.m. L-asparaginase, i.v. cylophosphamide, p.o. 6-thioguanine, i.v. or s.c. Ara-C and i.t. MTX), interim maintenance II for 2 months (same as interim maintenance I) and maintenance 12-week cycles (i.v. vincristine, p.o. dexamethasone, p.o. 6-mercaptopurine, p.o. MTX and i.t. MTX). Therapy was continued for 2 calendar years for girls and 3 calendar years for boys.

Summary of modified CCG 1961 protocol for high-risk ALL. Patients were eligible for this protocol if they had previously untreated ALL with $>25 \%$ blasts (L1 or L2 morphology) in bone marrow. Patients with FAB L3 were not eligible.

A) Standard arm. The standard arm included patients aged 1-9.99 years with initial $\mathrm{WBC}>50.000 / \mu 1$, patients aged $>10$ years with any WBC count, patients with overt testicular leukemia and patients with T-ALL. Patients in the standard arm received induction chemotherapy for one month (same as standard risk plus i.v. doxorubicin), consolidation therapy for 5 weeks (i.v. cylophosphamide, p.o. 6-mercaptopurine, i.v. or s.c. Ara-C and i.t. MTX), interim maintenance I for 2 months (p.o. 6-mercaptopurine, p.o. MTX and i.t. MTX), delayed intensification for 2 months (i.v. vincristine, i.v. doxorubicin, p.o. dexamethasone, i.m. L-asparaginase, i.v. cylophosphamide, p.o. 6-thioguanine, i.v. or s.c. Ara-C and i.t. MTX), interim maintenance II for 2 months (same as interim maintenance I) and maintenance 12-week cycles (same as standard risk treatment). Therapy was continued for 2 calendar years for girls and 3 calendar years for boys.

B) Augmented arm. The augmented arm included patients with CNS disease at diagnosis and patients with poor response at day 14 (both standard and high risk). Patients received induction chemotherapy for one month (same as standard risk treatment plus i.v. doxorubicin), consolidation for 9 weeks (i.v. vincristine, i.v. cylophosphamide, i.m. L-asparaginase, p.o. 6-mercaptopurine, i.v. or s.c. Ara-C and i.t. MTX plus cranial radiotherapy 18 Gy for patients without CNS disease at diagnosis and 24 Gy for those with CNS disease at diagnosis), interim maintenance I and II, each for 2 months (i.v. vincristine, i.v. MTX, i.m. L-asparaginase and i.t. MTX), delayed intensification I and II for 8 weeks (i.v. vincristine, i.v. doxorubicin, p.o. dexamethasone, i.m. L-asparaginase, i.v. cylophosphamide, p.o. 6-thioguanine, i.v. or s.c. Ara-C and i.t. MTX) and maintenance 12-week cycles (same as standard risk treatment). Therapy was continued for 2 calendar years for girls and 3 calendar years for boys.

Ethics. The study was performed in accordance with ethical standards and with the Helsinki Declaration of 1964, as revised in 2000. The study was approved by the local ethics committee and informed consent was obtained from the study participants.

Statistical analysis. Data were checked, entered and analyzed using SPSS version 11. Data are expressed as the mean \pm standard deviation for quantitative variables, and as a number and percentage for qualitative ones. Paired t-test and Chi-square 
Table I. Demographic, clinical and laboratory data of patients.

\begin{tabular}{|c|c|c|}
\hline Parameter & $\mathrm{n}$ & $\%$ \\
\hline \multicolumn{3}{|l|}{ Age at diagnosis (years) } \\
\hline Mean \pm SD & \multicolumn{2}{|c|}{$6.9 \pm 3.04$} \\
\hline Range & \multicolumn{2}{|c|}{$2.5-13$} \\
\hline \multicolumn{3}{|l|}{ Age at study (years) } \\
\hline Mean \pm SD & \multicolumn{2}{|c|}{$12.9 \pm 3.2$} \\
\hline Range & \multicolumn{2}{|c|}{$8.5-20$} \\
\hline \multicolumn{3}{|l|}{ Gender } \\
\hline Male & 14 & 56.0 \\
\hline Female & 11 & 44.0 \\
\hline \multicolumn{3}{|l|}{ Risk } \\
\hline SR & 15 & 60.0 \\
\hline HR & 10 & 40.0 \\
\hline \multicolumn{3}{|l|}{ Protocol of treatment } \\
\hline CCG-SR & 15 & 60.0 \\
\hline CCG-HR-SA & 6 & 24.0 \\
\hline CCG-HR-AA & 4 & 16.0 \\
\hline \multicolumn{3}{|l|}{ Immunophenotyping } \\
\hline Precursor B-ALL & 22 & 88.0 \\
\hline T-ALL & 3 & 12.0 \\
\hline \multicolumn{3}{|l|}{ CNS manifestations at diagnosis } \\
\hline Yes & 3 & 12.0 \\
\hline No & 22 & 88.0 \\
\hline \multicolumn{3}{|l|}{ Cranial irradiation } \\
\hline Yes & 4 & 16.0 \\
\hline No & 21 & 84.0 \\
\hline \multicolumn{3}{|l|}{ Late neurological complications } \\
\hline None & 23 & 92.0 \\
\hline Epilepsy & 1 & 4.0 \\
\hline $\begin{array}{l}\text { Cognitive changes, } \\
\text { behavioral changes, epilepsy }\end{array}$ & 1 & 4.0 \\
\hline
\end{tabular}

SR, standard risk; HR, high risk; CCG-SR, Children's Cancer Group - standard risk; CCG-HR-SA: Children's Cancer Group - high risk, standard arm; CCG-HR-AA, Children's Cancer Group - high risk, augmented arm; ALL, acute lymphoblastic leukemia; CNS, central nervous system.

$\left(\chi^{2}\right)$ tests were used when appropriate. $\mathrm{P}<0.05$ was considered to indicate a statistically significant result.

\section{Results}

Patient characteristics. The demographic, clinical and laboratory data of the patients are listed in Table I.

MRI findings of patients. Abnormal MRI findings were detected in six patients (24\%). The abnormalities were in the form of leukoencephalopathy in two patients (one had grade III and the other had grade I) (8\%), brain atrophy in two patients $(8 \%)$, old infarct in one patient $(4 \%)$ and old hemorrhage in one patient (4\%) (Table II).
Table II. MRI findings of patients $(n=25)$.

\begin{tabular}{lrr}
\hline MRI findings & $\mathrm{n}$ & $\%$ \\
\hline Normal & 19 & 76.0 \\
Leukoencephalopathy & 2 & 8.0 \\
Brain atrophy & 2 & 8.0 \\
Old infarct & 1 & 4.0 \\
Old hemorrhage & 1 & 4.0 \\
\hline
\end{tabular}

MRI, magnetic resonance imaging.
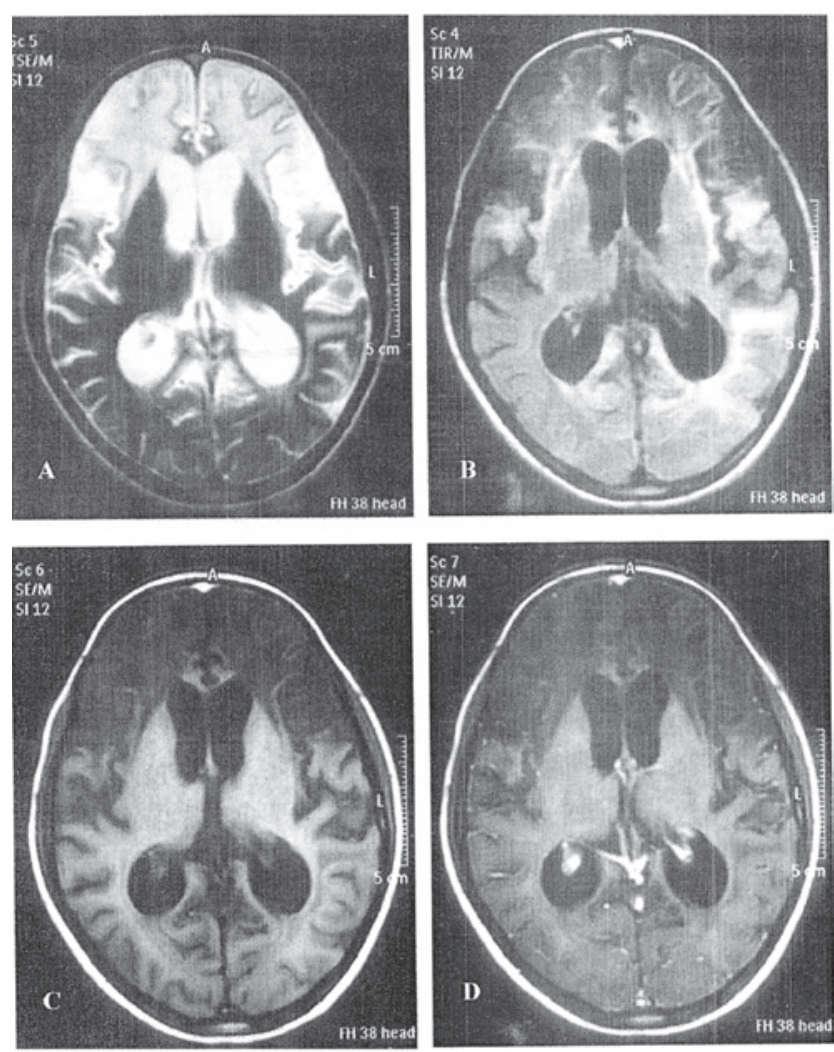

Figure 1. Magnetic resonance imaging of the brain of a 13-year-old boy previously treated with cranial irradiation. Diffuse bilateral confluent cortical and subcortical areas of abnormal attenuation are visible in both cerebral hemispheres, mainly in the frontal and parietal areas. High signal intensity was observed on (A) T2W1 and (B) FLAIR and low signal intensity was observed on T1W1 (C) without contrast and (D) with intravenous contrast. The images are consistent with grade III leukoencephalopathy. FLAIR, fluid attenuated inversion recovery; T2W, T2-weighted; T1W, T1-weighted.

Correlation between MRI findings and demographic data of patients. There was no significant correlation between MRI findings and the age and gender of patients $(\mathrm{P}=0.37$ and $\mathrm{P}=0.89$, respectively).

Correlation between MRI findings and immunophenotyping of leukemic cells. There was no significant correlation between MRI findings and immunophenotyping $(\mathrm{P}=0.75)$.

Correlation between MRI findings and risk group of patients. There was a significant correlation between MRI findings and 
Table III. Correlation between MRI findings and risk group.

\begin{tabular}{|c|c|c|c|c|c|c|}
\hline \multirow[b]{2}{*}{ Risk } & \multicolumn{2}{|c|}{ MRI normal $(n=19)$} & \multicolumn{2}{|c|}{ MRI abnormal $(n=6)$} & \multirow[b]{2}{*}{$\chi^{2}$} & \multirow[b]{2}{*}{ P-value } \\
\hline & $\mathrm{n}$ & $\%$ & $\mathrm{n}$ & $\%$ & & \\
\hline $\mathrm{SR}(\mathrm{n}=15)$ & 14 & 73.7 & 1 & 16.7 & 4.03 & $0.04^{\mathrm{a}}$ \\
\hline $\mathrm{HR}(\mathrm{n}=10)$ & 5 & 26.3 & 5 & 83.3 & & \\
\hline
\end{tabular}

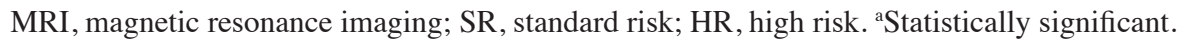

Table IV. Correlation between MRI findings and treatment protocol.

\begin{tabular}{|c|c|c|c|c|c|c|}
\hline \multirow[b]{2}{*}{ Protocol } & \multicolumn{2}{|c|}{ MRI normal $(n=19)$} & \multicolumn{2}{|c|}{ MRI abnormal $(n=6)$} & \multirow[b]{2}{*}{$\chi^{2}$} & \multirow[b]{2}{*}{ P-value } \\
\hline & $\mathrm{n}$ & $\%$ & $\mathrm{n}$ & $\%$ & & \\
\hline CCG-SR $(n=15)$ & 14 & 73.7 & 1 & 16.7 & & \\
\hline CCG-HR-SA $(n=6)$ & 5 & 26.3 & 1 & 16.7 & 15.31 & $<0.001^{\mathrm{a}}$ \\
\hline CCG-HR-AA $(n=4)$ & 0 & 0.0 & 4 & 66.6 & & \\
\hline
\end{tabular}

MRI, magnetic resonance imaging; CCG-SR, Children's Cancer Group - standard risk; CCG-HR-SA, Children's Cancer Group - high risk (standard arm); CCG-HR-AA, Children's Cancer Group - high risk (augmented arm). ${ }^{\text {a Significant. }}$

Table V. Correlation between MRI findings and CNS manifestations at diagnosis.

\begin{tabular}{|c|c|c|c|c|c|c|}
\hline \multirow{2}{*}{$\begin{array}{l}\text { CNS manifestations } \\
\text { at diagnosis }\end{array}$} & \multicolumn{2}{|c|}{ MRI normal $(n=19)$} & \multicolumn{2}{|c|}{ MRI abnormal $(n=6)$} & \multirow[b]{2}{*}{$\chi^{2}$} & \multirow[b]{2}{*}{ P-value } \\
\hline & $\mathrm{n}$ & $\%$ & $\mathrm{n}$ & $\%$ & & \\
\hline Yes $(n=3)$ & 0 & 0.0 & 3 & 50.0 & 6.58 & $0.01^{\mathrm{a}}$ \\
\hline No $(n=22)$ & 19 & 100.0 & 3 & 50.0 & & \\
\hline
\end{tabular}

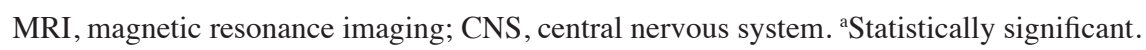

Table VI. Correlation between MRI findings and cranial irradiation.

\begin{tabular}{|c|c|c|c|c|c|c|}
\hline \multirow[b]{2}{*}{ Cranial irradiation } & \multicolumn{2}{|c|}{ MRI normal $(n=19)$} & \multicolumn{2}{|c|}{ MRI abnormal $(n=6)$} & \multirow[b]{2}{*}{$\chi^{2}$} & \multirow[b]{2}{*}{ P-value } \\
\hline & $\mathrm{n}$ & $\%$ & $\mathrm{n}$ & $\%$ & & \\
\hline Yes $(n=4)$ & 0 & 0.0 & 4 & 66.7 & 10.53 & $<0.001^{\mathrm{a}}$ \\
\hline No $(n=21)$ & 19 & 100.0 & 2 & 33.3 & & \\
\hline
\end{tabular}

MRI, magnetic resonance imaging. ${ }^{\text {aSignificant. }}$

risk group, where abnormal MRI findings were significantly higher in high-risk patients $(\mathrm{P}=0.04$; Table III).

Correlation between MRI findings and treatment protocol. There was a significant correlation between MRI findings and treatment protocol where abnormal MRI findings were significantly higher in patients who received CCG high-risk (augmented arm) protocol $(\mathrm{P}<0.001$; Table IV).
Correlation between MRI findings and CNS manifestations at diagnosis. There was a significant correlation between MRI findings and CNS manifestations at diagnosis where abnormal MRI findings were significantly higher in patients with CNS manifestations at diagnosis (Table V).

Correlation between MRI findings and cranial irradiation. There was a significant correlation between MRI findings 
and cranial irradiation where abnormal MRI findings were significantly higher in patients who received cranial irradiation $(\mathrm{P}<0.001$; Table VI).

Correlation between MRI findings and late neurological complications. There was no significant correlation between MRI findings and late neurological complications $(\mathrm{P}=0.37)$.

\section{Discussion}

Currently, the overall long-term survival rate in ALL is approximately $70 \%$, although for individual patients this varies from $40-90 \%$, depending on prognostic features at diagnosis and early response to therapy $(9,10)$. Previously, however, CNS relapse occurred in at least $50 \%$ of patients (11).

This phenomenon led to the introduction of CNS prophylaxis for children with ALL. The most commonly used method in the 1970s and 1980s involved cranial irradiation, originally at a dose of $24 \mathrm{~Gy}$ but later at $18 \mathrm{~Gy}$, and intrathecal chemotherapy with MTX (12). This treatment reduces the rate of isolated CNS relapse to $5-10 \%$ but is associated with various forms of damage to normal brain tissue, including leukoencephalopathy, mineralizing microangiopathy (MMA), and the development of secondary tumors (11).

Cranial irradiation appears to be a notable cause of long-term neuropsychological impairment (13). Protocols have used risk stratification to avoid cranial irradiation in children with standard and intermediate-risk $\operatorname{ALL}(14,15)$ and reduced the dose to 12 Gy in those with high-risk ALL, without compromising event-free survival (3).

In our study, abnormal MRI findings were detected in six patients (24\%). They were in the form of leukoencephalopathy in two patients (one had grade III and the other had grade I) $(8 \%)$, brain atrophy in two patients $(8 \%)$, old infarct in one patient $(4 \%)$ and old hemorrhage in one patient $(4 \%)$. The brain MRI of a 13-year-old boy with grade III leukoencephalopathy is shown in Fig. 1.

Our results are similar to those reported by Ficek et al (16) where white matter changes were detected by MRI in three (11\%) out of 45 ALL survivors treated between 1994 and 2002 and examined for 6-12 years following treatment. All children with MRI abnormalities received CRT.

Pääkkö et al (7) carried out a prospective study on 33 children with ALL and observed high-intensity white matter changes by MRI in 3 children (9\%) who received chemotherapy only.

Aytaç et al (17) reviewed the data of 256 children with ALL who were admitted to the Pediatric Hematology Unit of Hacettepe University, Turkey, between March 1991 and May 2005 and who were eligible for and treated according to the St. Jude Total XI and XIII protocols. Abnormal MRI findings were reported in only one out of five patients, for whom MRI was performed following cessation of treatment. The abnormalities were in the form of an increase in white matter intensity.

Chan et al (5) evaluated the brains of 42 patients diagnosed more than 5 years previously. Forty of the ALL patients had been treated with cranial irradiation (at least $18 \mathrm{~Gy}$ ) and intrathecal MTX, as well as systemic chemotherapy, and two had been treated with intrathecal MTX and systemic chemotherapy but had not received cranial irradiation. Lesions consistent with old hemorrhage were detected in 23 (55\%) of the ALL patients, white matter abnormalities were found in two patients $(5 \%)$ while old infarcts were observed in four patients (10\%). Lesions were observed in all 40 patients who underwent cranial irradiation.

Our results showed that there was no significant correlation between MRI findings and age, gender or immunophenotyping of leukemic cells.

Conversely, Pääkkö et al (7) reported that children with white matter changes were significantly younger than those with normal MRI (mean age 2.8 vs. 7.4 years).

In our study, cranial irradiation was administered to only four patients who received the augmented arm of the CCG high-risk protocol. Three of them were assigned to this arm based on their initial CNS infiltration and one was assigned based on his poor response to the standard arm of this high-risk protocol.

In our study, there was a significant $(\mathrm{P}<0.001)$ correlation between MRI findings and cranial irradiation. All patients who received cranial irradiation developed abnormal MRI findings while only two out of $21(9.5 \%)$ who received systemic chemotherapy and intrathecal methotrexate without cranial irradiation developed abnormal MRI findings. Our results augment and support the idea that cranial irradiation should be avoided in patients with standard risk criteria. In support of this theory, Chan et al (5) and Ficek et al (16) reported that all abnormal MRI lesions were observed in patients who underwent cranial irradiation.

Radiation-induced brain toxicity was explained by Kim et al (18) who found that radiation injures the supportive tissues and neurogenic microenvironment of the nervous system and leads to neuronal loss or damage. Oxygen-free-radical damage and altered cytokine responses may influence the development of late delayed damage. Glial and neuronal stem-cell damage may result in a progressive demyelination and/or neuronal cell loss.

In our study, there was a significant correlation between MRI findings and risk group $(\mathrm{P}=0.04)$ where five out of six patients who developed abnormal MRI findings belonged to the high-risk group and only one belonged to the standard risk group. Also, there was a significant $(\mathrm{P}<0.001)$ correlation between MRI findings and treatment protocol where the four patients $(100 \%)$ who received the CCG high-risk augmented arm protocol developed abnormal MRI findings while one out of $15(6.7 \%)$ who received the CCG high-risk standard arm protocol developed abnormal MRI findings and one out of six (16.7\%) who received the CCG standard risk protocol developed abnormal MRI findings. This finding can be attributed to the fact that patients who received the CCG high-risk augmented arm underwent cranial irradiation. In our study, there was a significant correlation $(\mathrm{P}=0.01)$ between MRI findings and CNS infiltration at diagnosis where all patients with CNS infiltration at initial diagnosis developed abnormal MRI findings and only three out of 22 (13.6\%) without CNS infiltration at diagnosis developed abnormal MRI findings. This can once more be attributed to patients with CNS infiltration at initial diagnosis receiving the CCG high-risk augmented arm protocol and undergoing cranial irradiation.

In our study, only two of our patients developed late neurological complications. One patient developed recurrent 
seizures and proved to have epilepsy. This patient belonged to the high-risk group and received the CCG high-risk standard arm protocol. He did not receive cranial irradiation and his MRI examination was completely normal. The second patient developed recurrent seizures, abnormal behavioral changes and severe cognitive changes. This patient belonged to the high-risk group and received CCG high-risk augmented arm protocol. He received cranial irradiation and he had grade III leukoencephalopathy by MRI examination.

The lower incidence (8\%) of late neurological complications in our study can be explained by the fact that most (84\%) of our patients did not receive cranial irradiation. Cranial irradiation is reserved for patients with CNS infiltration at initial diagnosis and for those with poor response to the standard risk protocol and the standard arm of the high-risk protocol. It can also be explained by the fact that $60 \%$ of our patients belonged to the standard risk group who received tolerable doses of chemotherapeutics.

In our study, there was no significant correlation between MRI findings and late neurological complications. This can be attributed to the small number of patients with late neurological complications.

We conclude that cranial irradiation is associated with higher incidence of MRI changes in children treated for ALL. Limitation of cranial irradiation to selected patients contributed to the lower incidence of neurological complications in our study. MRI is a sensitive radiological tool to detect structural changes in children treated for ALL, even in asymptomatic cases.

\section{References}

1. Pui $\mathrm{CH}$ and Evans WE: Treatment of childhood acute lymphoblastic leukemia. N Engl J Med 354: 166-178, 2006.

2. Tucci $F$ and Arico M: Treatment of pediatric acute lymphoblastic leukemia. Haematologica 93: 1124-1128, 2008.

3. Schrappe M, Reiter A and Ludwig WD: Improved outcome in childhood acute lymphoblastic leukemia despite reduced use of anthracyclines and cranial radiotherapy: Results of trial ALL-BFM90. German-Austrian-Swiss ALL-BFM study group. Blood 95: 3310-3322, 2000.

4. Pullen J, Boyett J, Shuster J, et al: Extended triple intrathecal chemotherapy trial for prevention of CNS relapse in good-risk and poor-risk patients with B-progenitor acute lymphoblastic leukemia: A Pediatric Oncology Group study. J Clin Oncol 11: 839-849, 1993.

5. Chan MSM, Roebuck DJ, Yuen MP, Li CK and Chan YL: MR imaging of the brain in patients cured of acute lymphoblastic leukemia - the value of gradient echo imaging. Am J Neuroradiol 27: 548-552, 2006.

6. Gay CT, Bodensteitter JB, Nitschke R, Sexauer C and Wilson D: Reversible treatment-related leukoencephalopathy. Child Neurol 4: 208-213, 1989.
7. Pääkkö E, Harila-Saari A, Vanionpää L, Himanen S, Pyhtinen J and Lanning M: White matter changes on MRI during treatment in children with acute lymphoblastic leukemia: correlation with neuropsychological findings. Med Pediatr Oncol 35: 456-461, 2000.

8. Koike S, Aida N, Hata M, Fujita K, Ozawa Y and Inoue T: Asymptomatic radiation-induced telangiectasia in children after cranial irradiation: frequency, latency, and dose relation. Radiology 230: 93-99, 2004.

9. Forestier E, Johansson B, Gustafsson G, Borgström G Kerndrup G, Johannsson J and Heim S: Prognostic impact of karyotypic findings in childhood acute lymphoblastic leukemia: a Nordic series comparing two treatment periods. Br J Haematol 110: 147-153, 2000.

10. Donadieu J and Hill C: Early response to chemotherapy as a prognostic factor in childhood acute lymphoblastic leukemia: a methodological review. Br J Haematol 115: 34-45, 2001.

11. Schroeder H, Garwicz S, Kristinsson J, Siimes MA, Wesenberg F and Gustafsson G: Outcome after first relapse in children with acute lymphoblastic leukemia: a population-based study of 315 patients from the Nordic Society of Pediatric Hematology and Oncology. Med Pediatr Onco1 25: 372-378, 1995.

12. Nesbit ME Jr, Sather HN, Robison LL, Ortega J, Littman PS, D'Angio GJ and Hammond GD: Presymptomatic central nervous system therapy in previously untreated childhood acute lymphoblastic leukaemia: comparison of $1800 \mathrm{rad}$ and $2400 \mathrm{rad}$ A report for Children's Cancer Study Group. Lancet 1: 461-466, 1981.

13. Hill JM, Kornblith AB, Jones D, et al: A comparative study of the long term psychosocial functioning of childhood acute lymphoblastic leukemia survivors treated by intrathecal methotrexate with or without cranial radiation. Cancer 82: 208-218, 1998.

14. Kamps WA, Bokkerink JP, Hahlen K, et al: Intensive treatment of children with acute lymphoblastic leukemia according to ALL-BFM-86 without cranial radiotherapy: results of Dutch Childhood Leukemia Study Group Protocol ALL-7 (1988-1991). Blood 94: 1226-1236, 1999.

15. Hill FG, Richards S, Gibson B, et al: Successful treatment without cranial radiotherapy of children receiving intensified chemotherapy for acute lymphoblastic leukaemia: results of the risk-stratified randomized central nervous system treatment trial MRC UKALL XI (ISRC TN 16757172). Br J Haematol 124: 33-46, 2004.

16. Ficek K, Blamek S, Syguła D, Miszczyk L, Sońta-Jakimczyk D and Tarnawski R: Evaluation of the late effects of CNS prophylactic treatment in childhood acute lymphoblastic leukemia (ALL) using magnetic resonance spectroscopy. Acta Neurochir Suppl 106: 195-197, 2010.

17. Aytaç S, Yetgin S and Tavil B: Acute and long-term neurologic complications in children with acute lymphoblastic leukemia. Turk J Pediatr 48: 1-7, 2006.

18. Kim JH, Brown SL, Jenrow KA and Ryu S: Mechanisms of radiation-induced brain toxicity and implications for future clinical trials. J Neurooncol 87: 279-286, 2008. 